\title{
Design of residential environments for people with dementia and sight loss: a
}

structured literature review

To be published in British Journal of Visual Impairment.

Cite as: Bowes A, Dawson A, Greasley-Adams C and McCabe L Design of residential environments for people with dementia and sight loss: a structured literature review (Forthcoming), British Journal of Visual Impairment.

Authors: Alison Bowes, Alison Dawson, Corinne Greasley-Adams, Louise McCabe

\section{Abstract}

A structured literature review concerning the design of living environments for people with dementia and sight loss was conducted. Following systematic searching, thirty three items were included and quality assessed. Findings are described covering colour and contrast, lighting, fixtures and fittings, entrances and exits, gardens and outdoors. The discussion highlights the poor quality of evidence, combined nevertheless with useful suggestions for design; the tendency for literature to be fragmented; and the need for improvements in terms of study focus, study quality and an emphasis on independence and individual needs. The review was subsequently used to inform the development of design guidelines.

\section{Keywords}

Design, dementia, Alzheimer's, sight loss, literature review 


\section{Background and aims}

A growing literature focuses on how environmental design can either enhance or inhibit independence for people with impairments. This includes research on design for people with sight loss and design for people with dementia, and design guidance which has been produced for each of these groups.

Advancing age is a well evidenced risk factor for sight loss as well as for dementia. Dementia can compound and complicate sight loss, as memory and understanding may become impaired.

Furthermore, some forms of dementia may entail physical damage which can produce additional sight problems. Despite this, researchers have been slow to consider the impairments together, and the scientific literatures have tended to remain largely separate.

Existing guidance also appears to have some limitations. Reviewing selected design guidance publications for people with sight loss and people with dementia, Goodman and Watson $\left(2010 \mathrm{~L}^{1}\right)$ suggest that, whilst there are many similarities, there appear to be some areas of potential conflict: for example, recommendations of curved paths and corridors for people with dementia and straight ones for people with sight loss (2010: 4) and recommendations of multiple objects placed around environments for people with dementia to support reminiscence and reduced clutter for people with sight loss (2010: 4). Furthermore, they identify that the evidence base for the guidance is not always clear, and they raise reliability issues.

Given the common co-occurrence of dementia and sight loss for older people, the apparent contradictions in existing guidance, and the questioning of the evidence base, this paper reports on a literature review of research on design of living environments for people with dementia and people with sight loss, which aimed to explore the evidence base for design recommendations. The literature review was subsequently used to inform the development of new design guidelines for people with dementia and sight loss (Greasley-Adams et al 2014). 


\section{Methods}

The structured literature review involved systematic searches conducted in December 2012 of a wide range of databases, complemented by consultation with expert informants who were asked to recommend materials (such as reports) that the searches might not have identified. Table $1 \mathrm{~S}$ (Supplement) lists the search terms used, Table 2S (Supplement) lists the electronic databases searched, Figure 1 (Supplement) summarises the results of the searches and the process of identifying relevant materials for review, and Table 3S (Supplement) provides the scoring system used as a final filter to select items for full text review. These were systematically evaluated using a proforma which enabled assessment of the quality of the research reported, and assembled key information regarding the subject matter, results and conclusions of each item. Reviewers identified the research design of the item being reviewed, and then responded to a series of questions relating to that specific research design. Table 4S (Supplement) lists the quality criteria used to assess different types of research. These were drawn from standard protocols widely used in reviewing, including Centre for Research and Development (CRD) Report No 4, used for Randomised Controlled Trials ; Cochrane Effective Practice and Organisation of Care (EPOC) checklists used for Controlled Before and After studies ; and Critical Appraisal Skills Programme (CASP) assessment criteria, used for Literature reviews and qualitative studies (NHS CRD 2001; Cochrane EPOC 2002; CASP undated). In addition, a section for 'other types of study' sought information about design and quality of these in a structured manner. Having responded to detailed questions about the design of the study, its conduct and conclusions, reviewers were asked to rate it as of High, Medium or Low quality and to record their reasons for doing so. Table 5S (Supplement) lists all the evaluations and the reviewers' summary reasoning. Thus each study was quality assessed according to specific criteria relating to studies using the same approach, and assessments were therefore structured and consistent within study type. A total of thirty three items were included in the review. Twenty-eight of these were identified by the searches and met the 
inclusion criteria, i.e. they focused on how people engaged with environments, described or reviewed a number of research studies, and related to people with dementia, people with sight loss and/or people with both. A further five items included were suggested as relevant by the panel of experts..

Following review, a descriptive and qualitative analysis of the findings was conducted. This was guided by design themes which are used to guide the presentation of findings. The themes were identified through consultations with 19 people experiencing dementia and/or sight loss and ten staff who worked with them: the consultations reviewed initial findings, and helped inform the thematic analysis of the findings. A full account of the consultations and the development of the guidelines, which took place throughout the process is given in (ref). A flowchart of the process of the literature review demonstrating where consultation was used is provided in the Supplement (Figure 2S).

\section{Quality and coverage of literature}

Table 1 summarises the nature of the literature evaluated in the review and indicates the quality assessments.

Table 1: Included items and quality assessments

\begin{tabular}{|l|l|l|l|l|}
\hline \multirow{2}{*}{ Type of study } & \multicolumn{4}{|l|}{ Quality assessment (number of items) } \\
\cline { 2 - 6 } & High & Medium & Low & Total \\
\hline Controlled before/after study & & 1 & & 1 \\
\hline Randomised controlled trial & & 1 & 2 & 3 \\
\hline Literature review & 3 & 3 & 3 & 9 \\
\hline Qualitative study & 2 & 3 & 3 & 8 \\
\hline Other & 2 & 1 & 11 & $12 *$ \\
\hline
\end{tabular}




\begin{tabular}{|l|l|l|l|l|}
\hline Total & 5 & 9 & 19 & 33 \\
\hline
\end{tabular}

* Other = enquiry report; practice guidelines (3); case study (2); cross-sectional population study; experiment; ideas review; product review; 3 study review; expert views.

Overall, there was little high quality research, consisting of three literature reviews and two qualitative studies. This means that our discussion can produce only indicative conclusions. Unlike some review procedures, we do not exclude lower quality work: especially in a new field of enquiry, this may provide useful indicative material. This contrasts with for example Anttila et al $(2012 \mathrm{H})$, whose review of the evidence base for assistive technology (AT) interventions for people with disabilities only included systematic reviews. We note that the strict inclusion criteria used by Martin et al $(2009 \mathrm{H})$ produced no relevant studies about smart home technologies, despite initially identifying 2,380 citations.

Table 2 indicates the populations covered in the literature. It is notable that the searches identified very little work relating to populations with both dementia and sight loss, supporting the need to focus specifically on this group in future research.

Table 2: Literature relating to which populations

\begin{tabular}{|l|l|}
\hline Population included & Number of items \\
\hline People with dementia or cognitive impairment & 13 \\
\hline People with sight loss or visual impairment & 12 \\
\hline People with both dementia/cognitive impairment & 3 \\
and sight loss/visual impairment & \\
\hline Other* & 5 \\
\hline
\end{tabular}

*Other = 'people of all ages with all disabilities', 'older people' (3), 'cognitive impairment, physical disability or learning disability'. 
Particular types of dementia or sight loss were rarely distinguished, with only two studies identifying type of dementia (both Alzheimer's disease) and only one specifying a single type of sight loss (age related macular degeneration) and one identifying a specific group of conditions (retinitis pigmentosa, diabetic retinopathy and glaucoma).

The large majority of sources focused on private spaces, including general housing stock, care homes or sheltered/extra care housing. There was a particular emphasis on care homes, with 17 items covering these. Where public buildings were considered, sources tended to cover a wide range, considering in general terms how places that people visited outside their homes could be more accessible by design.

Table 3 lists the wide range of design features considered and Table 4 the design properties.

Table 3: Design features covered in the literature

\begin{tabular}{|l|l|}
\hline Design feature & Number of items that \\
\hline particular room) & 11 \\
\hline Architectural aspects of a building (e.g. & \\
arrangements of rooms, corridors etc) & 11 \\
\hline Internal fixtures and fittings (e.g. switches, baths, & 12 \\
toilets) & \\
\hline Internal finishes (e.g. wall textures, paint types) & 12 \\
\hline Furniture and furnishings (e.g. wardrobes, & 17 \\
drawers, carpets) & \\
\hline
\end{tabular}




\begin{tabular}{|l|l|}
\hline Outside areas attached to buildings (e.g. gardens, & 11 \\
driveways) & \\
\hline Other features** & 8 \\
\hline
\end{tabular}

*More than one design feature was frequently covered in one publication

** Other = the whole building (2); technologies (2); lighting (2); neighbourhood design (1); multiple elements (1)

Table 4: Design properties addressed in the literature

\begin{tabular}{|l|l|}
\hline Design properties & Number of items that \\
& addressed this* \\
\hline Colour & 11 \\
\hline Texture (fabrics, wall coverings, hand rails, & 6 \\
\hline Controls) & \\
\hline Lighting levels & 11 \\
\hline Other & 18 \\
\hline
\end{tabular}

*More than one design property was frequently covered in one publication

**Other = assistive technology (3), smart home technology, features of nature and gardens, green environments, aspects of gardening that appeal to the different senses, glare, sound minimising, visual cues and use of space, visual barriers and distractions, automated verbal cues, multiple elements (2) 


\section{Findings of literature review}

We summarise the findings of the literature review under the headings that emerged from the thematic analysis of finding.

\section{Colour and contrast}

Research relating to colour and contrast was mainly of low quality, but a number of consistent findings and recommendations did emerge. The balance of views suggested that contrast rather than colour per se was of particular significance.

For example for people with dementia, Goodman and Watson (2010L) suggest using colour to define spaces, such as those for dining, relaxing or activity areas. Marquardt (2011M) supports this by suggesting that vivid colour coding might help improve functional ability and short term-memory, since people with dementia may have impaired ability to discriminate colour. Kelly et al (2011M) suggest that use of colour can aid way-finding and enhance visibility of features. . Calkins (2002L) argues that colour should be used to emphasize what is important (e.g. signage, orientation cues, stair edges and level changes, etc.) and de-emphasize that which is not. She suggests using brighter colours and higher contrast with background, adding that varying colour value by at least two levels on a 10-level scale will help people with dementia to distinguish between colours.

Kelly et al (2011M) suggest that in care homes, strong contrasting colours can help draw attention to areas that will be used by residents. For example, contrasting doors and doorframes might be beneficial, as might toilet seats that contrast with toilets. Other lower quality examples include McNair et al (2010L), who employ the same principle when suggesting that dining rooms in care homes for people with dementia should 'use white dishes with a contrasting coloured dark rim'. Calkins (2002L) suggests the use of contrasting colours may be helpful in a range of settings, including: chair seats; sinks and washstands; for toilets or toilet seats; and for table settings, using pale plates on darker tablecloths. 
High contrast markers may also help people with dementia identify hazards (Lawrence and Murray 2009L).

Contrast may also present difficulties. For example Goodman and Watson (2010L) call attention to the potential for sudden changes in contrast at floor level to be seen by some as a barrier and potentially to discourage movement. They note that some guidance considers the use of such contrast to deter people from entering certain spaces, arguing that the ethical aspects of such measures require careful attention: they could be seen as a form of restraint.

For people with sight loss, good contrasts are reported to be important (Brunnström et al 2004L) and contrasting colours can aid object recognition (Unwin et al 2009L). For example, pull cords for blinds, and light switches could be high contrast with surroundings (Littlefair 2009L) and chairs could be contrasted with walls to help people see them (Torrington and Lewis 2011M). Littlefair (2009L) suggests that light coloured window frames and window walls reduce contrast between the window and its surroundings and might help in reducing glare. However, Cooper (1999M) found that people disagreed that they needed supports using colour and contrast.

Den Brinker et al (2005M) and Unwin et al (2009L) suggest that for people with sight loss, contrasting strips placed on the edge of stair-treads can help reduce the risk of falls. Littlefair (2009L) suggests that lighting which creates contrast between tread and risers on stairways and which casts shadows can help to highlight the three-dimensional form of stairs and adds that natural lighting from the side is best for stairways. East or west facing windows at the top and bottom of stairs should be avoided due to increased risk of glare from the rising or setting sun (Goodman 2008, referred to in Littlefair 2009L).

For people with both dementia and sight loss, Lawrence and Murray (2009L) recommend using bold colour and contrast to aid vision and independence: for example, they may assist with identifying objects and distinguishing them from their background more easily. 


\section{Lighting}

Several low and medium quality publications refer to the need for adequate lighting levels in home environments for people with dementia (e.g. Calkins and Brush Calkins 2002L; McNair et al 2010L; van Hoof et al 2010M; Kelly et al 2011M; Marquardt 2011M). McNair et al (2010:9L) argue that there is a clear link between appropriate lighting and supporting independence.

The type of light may be important, though evidence in this area is not strong. Natural light is said to have beneficial properties for people with dementia, including improving health and wellbeing (Bossen 2010L), lowering incidence of falls and sleep disturbance (McNair et al 2010L) and assisting with way finding and thus independence (Marquardt 2011M). For people with dementia living in care homes, Marquardt (2011M) argues that sensory stimulation through natural light and views to the outside are beneficial, and long internal corridors lacking this will negatively affect vitality.

Several studies find benefits from bright light. Marquardt (2011M) points to studies which have demonstrated that bright artificial lighting and natural light can improve sleep duration, reduce agitation and aggressive behaviour and assist with way-finding. Lawrence et al's (2009H) high quality work which also focuses on care homes, suggests that dim lighting is associated with challenging behaviour in people with dementia. Van Hoof et al's (2010M) literature review notes that improving lighting is often mentioned as a means to increase safety and poor lighting has been identified as an environmental hazard in the homes of older people. They suggest that good lighting can increase overall quality of life and prevent falls; it might be able to help modify behavioural symptoms and improve cognitive function; it can make hazards more visible; and in dining rooms, it can enable people to eat better.

Lighting recommendations for particular rooms for people with dementia are made. In bathrooms for people with dementia McNair et al (2010L) suggest: using multiple ceiling mounted lights to ensure no dark corners; providing task lighting at wash-basins; using light-transmitting material for shower cubicles 
or mounting an appropriate ingress-protected light directly above the cubicle; and using a movement detector at the door to turn lights on automatically. However, Van Hoof et al (2010M), with stronger evidence caution that automatic lights switching on can cause distress to people with dementia.

In kitchens, McNair et al's (2010L) specific suggestions include: using long light sources (i.e. fluorescent tubes) for general lighting rather than spotlights; low profile strip lighting under cabinets for task lighting, protected from direct view by pelmets; similar lighting on top of wall mounted cupboards to avoid dark areas, protected from direct view by cornices; and providing specific task lighting over the sink. Similarly, Van Hoof et al (2010M) mention the need for greater task illumination for food preparation.

For people with sight loss, lower quality research such as Littlefair (2009L) reports that natural day lighting is generally more comfortable than electric task lighting. Long (2005L) supports this, reporting that people with sight loss acknowledge the desirability of natural light throughout their homes. Variation of natural daylight can help with sleep and add to interest in the visual environment; and windows can help with way finding (Littlefair 2009L).

Poor lighting can compromise home safety for people with sight loss, whereas good lighting can bring benefits beyond simply improved safety. In a randomised controlled trial of a home safety intervention for people with visual impairment, La Grow et al (2006L) found that nearly 40\% (77 of 194) homes visited presented safety hazards related to indoor lighting. Calkins and Brush (2002L) and Kelly et al (2011M) note that care homes also tend to fall short in these aspects. Brunnström et al (2004L) found that having good quality lighting in a living room can benefit quality of life and that improving the quality of lighting in bathrooms and kitchens could lead to improvements in activities of daily living (ADLs). This is supported by van Hoof et al (2010M), who note that people with visual impairments may have impaired ability to adapt to changing light conditions. 
These recommendations differ from other literature relating to lighting for people with sight loss which highlights the preferences of people themselves, and their individual requirements. For example, Littlefair (2009L) notes that people with visual impairments may appreciate a degree of variation in light in rooms and will organise tasks according to lighter or darker areas. Similarly Long (2005L) points out that people with sight loss have unique lighting requirements depending on the type of vision loss, sensitivity to glare and the task being undertaken. His study emphasises both the need to increase sources of natural and artificial lighting in people's homes, and to be able to control lighting. Increased availability of lighting could include: strip lighting under kitchen cabinets; lights within shower areas; well-lit exterior doors and lighted keyholes (see also Unwin et al 2009L) and sound- or motion-activated external entrance lighting. Increased control could include the use of rheostats on all light switches; adjustable blinds; and tinted large windows/skylights to reduce glare. Long (2005L) and Percival (2007M) both argue that control of lighting is particularly important for people with sight loss - more so than colour and contrast - and is relatively simple to achieve.

Considering kitchens for people with sight loss, Long (1995L) discusses the need for additional lighting including strip lighting under cabinets (a recommendation also supported by Unwin et al 2009). Brunnström et al (2004L) found that providing ceiling-mounted fluorescent tube light fittings together with under-cupboard lighting on working surfaces led to significant improvement in ADLs for tasks carried out on the working surface in the kitchen. Littlefair (2009L) however noted the preference of people with visual impairments for daylight in kitchens, especially for task areas.

For people with both sight loss and dementia, Goodman and Watson (2010L) state that there is a need to maximise natural light, supported by the Homes and Communities Agency (2009L). Edgerton and Ritchie (2010L) suggest that lighting should be at a level that helps to compensate for poor eyesight in people with dementia to aid with way finding. Goodman and Watson (2010L) also suggest that stark 
differences in lighting levels, such as areas of excessive light and shadowed areas, should be avoided. They too highlight the need to be able to adapt or adjust the lighting levels to suit individual needs. The need to couple appropriate intensity of light with good contrast conditions and absence of glare has been noted both for those with sight loss (Brunnström et al 2004L) and for people with dementia (McNair et al 2010L).

The many recommendations about lighting made in this literature are not wholly consistent, and not generally well evidenced. Nevertheless, it is possible to identify the importance of individual choice and the ability to control one's environment by, for example, adjusting lighting according to one's own preferences.

\section{Fixtures and fittings}

The literature makes multiple recommendations about fixtures and fittings, often in relation to particular rooms in the house, notably kitchens and bathrooms. Under this heading, there was relatively little literature relating specifically to people with dementia and most of the recommendations relate to people with sight loss. The quality of this literature is not generally high.

In care homes, Calkins and Brush (2002L) discuss the use of soft furnishings such as curtains and acoustic panels to absorb noise which might otherwise distract diners in dining rooms. Edgerton and Richie's (2010L) brief literature review suggested creating the perception of choice for care home residents with dementia by using low level dividers or partitions to create smaller spaces in larger rooms, research having shown that providing choice could improve quality of life and have a calming influence on residents.

In people's own homes, Unwin et al (2009L) present a list of potential modifications which might allow people with dementia to remain there for longer. Many of these relate to fixtures and fittings including: grab rails in the bathroom; room-darkening curtains in the bedroom; bannisters on both sides of stairs; 
and removing rugs without non-skid backing. Kiata et al (2008L) find that people do not necessarily want to remove rugs, preferring to protect the carpets underneath.

For people with dementia, fixtures and fittings in bathrooms are encouraged to be visible, using contrast (Kelly et al 2011M). Unwin et al (2009L) warn of a potential issue regarding mirrors in bathrooms for people with dementia, who may not recognise their reflections and find this distressing: this presents a contrast with recommendations for people with sight loss (Long 1995L) to have an accessible mirror available.

Regarding people with sight loss, several points made about fixtures and fittings relate to: the provision of hand rails or grab rails (Dahlin-Ivanoff and Sonn 2004M; La Grow et al 2006L); the use of rugs and mats (La Grow et al 2006L; Kiata 2008L); electrical outlet sockets and switches (Long 1995L); appliance controls generally (Long 1995L; Kondo et al 1997L; Littlefair 2009L) and kitchen cupboard doors and handles (Long 1995L). More generally, Lawrence and Murray (2009L) refer to bold colour and contrast in fixtures and fittings as a means of promoting independence through aiding vision.

Goodman and Watson (2010L) suggest that in respect of fixtures and fittings there may be different requirements for people with dementia and people with sight loss and make several related points. They note firstly fittings and equipment made or adapted for people with sight loss may need to incorporate less 'traditional' and less familiar types of controls (e.g. more tactile controls). Secondly, the 'old fashioned', more familiar, design of fittings and fixtures often recommended for home environments for people with dementia may promote an 'institutional' image that accommodation providers for people with sight loss would be keen to avoid and which might deter interaction/visits from the wider community. Thirdly, fixtures and fittings which assist with reduced functional abilities often experienced by people further into their dementia journey would not be necessary in homes of people with sight loss. Fourthly, some items suggested as providing memory stimulants might present hazards to people 
with sight loss (they use the example of a traditional clothes lines with stretchers). Mihailidis and Fernie (2002L) identify that assistive technology that can respond to individual requirements and wishes is increasingly feasible, suggesting increasing potential for flexibility.

\section{Entrances and exits}

The literature on entrances and exits relating to people with dementia tends to emphasise control. For example, Edgerton and Richie (2010L) suggest that unobtrusive and disguised exits and safety features can reduce 'exit attempts' by people with dementia. Letts et al $(2011 \mathrm{H})$ also comment on how visual barriers are effective in reducing attempts at exiting. To facilitate people using entrances and exits, Kelly et al (2011M) again suggest that strong contrasting colours, such as between doors and door frames can help draw attention to areas that will be used by care home residents.

Control of use of exits, whether deliberate or inadvertent, can have unintended consequences. For example, where difficulties exist in accessing gardens, and where help is required to access such spaces there will be reduced use of them: Rappe and Topo $(2007 \mathrm{H})$ suggest that easy access promotes daily use. Bossen (2010L) notes a number of potential barriers to the use of outdoor spaces by residents of care homes for people with dementia, including: heavy or locked doors; entrance or exit designs which fail to facilitate disabled access; lack of cueing features or landmarks; and lack of weather protection. Kwack et al (2005L) mention the roles of raised door sills and poorly located exits to gardens in deterring the use of outdoor spaces.

By contrast, literature on entrances and exits for people with sight loss emphasizes their ability to use these independently. For example, Long (1995L) suggests that larger than normal landings at stairs makes their use easier for people with sight loss; it is desirable for people with sight loss not to have proud door thresholds, as these may be a tripping hazard; pocket or sliding doors, and recessed door handles reduce the risk of walking into doors; well-lit exterior doors and lighted keyholes, as well as 
sound-activated light can be beneficial to those with sight problems; alarm control panels should have tactile markings and audible confirmation when buttons are pressed; and intercoms should be positioned to allow doors to be entered from anywhere in the property. Unwin et al (2009L) also suggest that people with sight loss might benefit from bright lights at external doors, and lighted keyholes.

Best and Porteous ${ }^{2}$ (2012L) assert that there should be straightforward access to buildings and that doorways should be built to wheelchair standards: this perspective emphasizes access and independence for all. Goodman and Watson (2010L), similarly, suggest that entrances and exits should be easy to locate and use by all.

\section{Gardens and outdoors}

People with dementia living in care homes who use gardens on a regular basis may experience benefits: Bossen's (2010L) review of literature on nature and nature-based stimuli for people with dementia indicates that amongst other benefits there is evidence that more frequent 'wander garden' users have fewer falls and their dosage of anti-psychotic medications may reduce.

Recommendations for gardens appropriate for people with dementia suggest they should be designed for accessibility to help people find features and to prevent injury. This might include using easy entrances, incorporating raised flowerbeds or container gardening, or pulleys to raise/lower hanging baskets, and adapting tools to make them easier to use (Kwack et al 2008L).

Recommendations are given for garden boundaries, generally for safety reasons (Kwack et al 2008L; Rappe and Topo 2007H; Unwin et al 2009L). For further safety within the garden, Kwack et al (2008L) suggest that contrasting colours to highlight features and hazards can help people with dementia and may reduce falls, hence preventing injury; Unwin et al (2009L) recommend the removal of all poisonous plants. 
To make the garden more pleasurable, Kwack et al (2008L) recommend that attention is given to the colours, scents and textures of plants to ensure olfactory, auditory and tactile stimulation. They suggest that plants should be selected in reference to the experiences, memories and preferences of residents. They recommend looped paths for people with dementia, to help guide their movement round a garden: however, Marquardt (2011M) suggests that continuous paths may interfere with way finding.

External steps or stairs, often located in or leading to gardens and outdoor areas, were identified as one of a range of potential hazards as part of a home safety program intervention trialled by La Grow et al (2006L). Steps, stairs, and kerbs were most commonly highlighted, accounting for $30 \%$ of all hazard related falls. A home safety programme addressing these issues was able to reduce falls.

Mitchell and Burton (2010M) argue that if local neighbourhoods are not dementia-friendly by design, people with dementia are likely to become increasingly isolated in their homes. Their work defines dementia-friendly neighbourhoods as welcoming, safe, easy and enjoyable for people with dementia and others to access, visit, use and find their way around. They identify six design principles: familiarity, legibility, distinctiveness, accessibility, comfort and safety and provide detailed recommendations as to how these could be achieved.

Considering people with sight loss and dementia, Goodman and Watson (2010L) observe that there may be differences in preferences for people with dementia and people with sight loss in respect of path layout, whereby straight paths with right angle turns where necessary are easier for people with sight loss than the looped paths recommended by some authors (e.g. Kwack et al 2008L).

\section{Discussion and conclusion}

The literature considers a wide range of design issues, with an emphasis on making recommendations either for people with dementia or for people with sight loss. Whilst the quality of research is not high, some relatively consistent recommendations can be identified as we have noted. Our subsequent work 
with service providers and service users in developing our guidelines (Greasley-Adams et al 2014) suggested that many of them made sense in the context of their experiences. However, it remains the case that the evidence base needs further research to build it more securely, as much of the literature is not research based, or has an unclear evidence base. There are widespread calls for further research, especially in terms of improved research design and scale of studies. Currently, research is often fragmented, focusing on small issues out of context and on single conditions, rather than on the whole person who is likely to experience several conditions, including dementia and sight loss. It is notable that we did not identify studies which had explicitly used research designs guided by the MRC framework for evaluating complex interventions (Craig et al 2008), recent developments in process evaluation (Moore et al 2015), or by innovative evaluation designs and approaches such as realistic evaluation (Pawson and Tilley 1997). Given that design issues are complex and do involve the whole person, exploring the use of such approaches appears merited.

There are some interesting contrasts in the literature relating to people with dementia and that relating to people with sight loss. For people with dementia, there is an emphasis on care settings, especially care homes. There are also a number of areas in which a key concern is control, such as the work on entrances and exits, and on altering behaviour, such as the recommendations about lighting to change people's sleep patterns, or activities to improve well-being, such as the use of gardens. We would not suggest that the emphasis on control and behaviour change is necessarily detrimental, but would urge careful consideration of the ethical aspects of the recommendations (cf. Zwijsen et al 2011M).

By contrast, the research relating to people with sight loss is focused on supporting independence and enabling people to do things for themselves: this is the 'default' for this research. We are aware that many of the design recommendations for people with dementia are derived from those for people with sight loss, and that many of them do indeed support independence (such as ease of eating at a table or 
using a bathroom). A consistent 'default' focus on independence for people with dementia would be welcome.

One stimulus for the present research, including this literature review, was the existence of apparent contradictions between design guidelines for people with dementia and those for people with sight loss: examples included, as we noted, debates about curved or straight paths and about reducing clutter or introducing reminiscence objects. If a genuinely person-centred perspective is adopted by service providers, whereby the needs of the individual are prioritized, there is a sense in which these are not contradictions, but simply alerts to a service provider to consider what is appropriate for particular individuals. Thus a core topic for future research must concern how to deliver person-centred design which has a clear evidence base.

\section{Declaration of conflicting interests}

The Authors declare that there are no conflicts of interest.

\section{Notes}

${ }^{1}$ The letters $\mathrm{H}$ (igh), $\mathrm{M}$ (edium) and $\mathrm{L}(\mathrm{ow})$ attached to publication dates report the quality assessments from the literature review. The process of quality assessment is described under 'Methods'.

${ }^{2}$ This work relates to older people in general and not specifically to people with sight loss and/or dementia. It was included in the review on the recommendation of one of our panel of experts. 


\section{References}

Anttila, H., Samuelsson, K., Salminen, A. \& Brandt, A. (2012). Quality of evidence of assistive technology interventions for people with disability: An overview of systematic reviews. Technology \& Disability, 24(1), 9-48.

Best R. \& Porteus J. (2012) 'Housing our Ageing Population: Plan for Implementation. HAPPI2', Report of the All Party Parliamentary Group on Housing and Care for Older People. Retrieved from http://www.housinglin.org.uk/ library/Resources/Housing/Support materials/Other reports and guid ance/Housing our Ageing Population Plan for Implementation.pdf .

Bossen, A. (2010). The importance of getting back to nature for people with dementia. Journal of Gerontological Nursing, 36(2), 17-22.

Bowes, A., Dawson, A., Greasley-Adams, C. \& McCabe, L. (20xx) .Developing best practice guidelines for designing living environments for people with dementia and sight loss. (under review)

Brunnström, G., Sörensen, S., Alsterstad, K. \& Sjöstrand, J. (2004). Quality of light and quality of life--the effect of lighting adaptation among people with low vision. Ophthalmic and Physiological Optics, 24(4), 274-280.

Brush, J.A., Meehan, R.A. \& Calkins, M.P. (2002). Using the environment to improve intake for people with dementia. Alzheimer's Care Quarterly, 2002; 3(4):330-8.

Calkins, M. P. (2002). How colour throws light on design in dementia care. Journal of Dementia Care, 10(4), 20-23. 
Calkins, M.P. (2002). Design a better bathroom: relaxing and comforting. Journal of Dementia Care, 10(3), 26-28.

Calkins, M.P. \& Brush, J.A. (2002). Designing for dining: the secret of happier mealtimes. Journal of Dementia Care, 10(2), 24-26.

Cochrane Effective Practice and Organisation of Care (EPOC) group (2002) 'Data Extraction Form, part of the EPOC Data Collection Template' [document on the internet]. July 2002 [cited 2013 Nov 12]. Available at http://epoc.cochrane.org/sites/epoc.cochrane.org/files/uploads/Data\%20Abstraction\%20Form\%20\%20EPOC\%20-\%202012-Feb-23.doc , but please note that this resource was updated in August 2013. Now see: Effective Practice and Organisation of Care (EPOC) (2015). EPOC Resources for review authors. Oslo: Norwegian Knowledge Centre for the Health Services. Available at: http://epoc.cochrane.org/epoc-specific-resources-review-authors, accessed 26 October 2015.

Cooper, B. A. (1999). The utility of functional colour cues: seniors' views. Scandinavian Journal of Caring Sciences, 13(3), 186-192.

Craig, P., Dieppe, P., Macintyre, S., Michie, S., Nazareth, I. and Petticrew, M. (2008) Developing and evaluating complex interventions: the new Medical Research Council guidance. British Medical Journal, $337,979-983$.

Critical Appraisal Skills Programme (CASP) (undated). 'Critical appraisal checklists' [document on the internet]. No longer available, but please note that resource was updated in 2013. Now see: Critical Appraisal Skills Programme (CASP) (2013). CASP Checklists. Available at http://www.casp-uk.net/\#!casptools-checklists/c18f8, accessed 26 October 2015.

Dahlin-Ivanoff, S. \& Sonn, U. (2004). Use of assistive devices in daily activities among 85-year-olds living at home focusing especially on the visually impaired. Disability \& Rehabilitation, 26(24), 1423-1430. 
den Brinker, B.P.L.M., Burgman, L.J., Hogervorst, S.M.J., Reehorst, S.E., Kromhout, S. \& van der Windt, J. (2005). The effect of high-contrast marking of treads on the descent of stairways by low-vision people. International Congress Series, 1282(0), 502-506.

Edgerton, E. A., \& Richie, L. (2010). Improving physical environments for dementia care: making minimal changes for maximum effect. Annals of Long Term Care, 18(5), 43-45.

Goodman, C. (2008). Housing for people with sight loss. Bracknell: Thomas Pocklington Trust/BRE Press.

Goodman, C., \& Watson, L. (2010). Design guidance for people with dementia and for people with sight loss, Thomas Pocklington Trust Research Findings Number 35. Retrieved from http://www.pocklingtontrust.org.uk/research/latest/rf35 (and full report available on request from Thomas Pocklington Trust).

Greasley-Adams, C., Bowes, A., Dawson, A. \& McCabe, L. (2014). Best Practice in the Design of Homes and Living Spaces for People Living with Dementia and Sight Loss. Stirling and London: Dementia Services Development Centre and the Thomas Pocklington Trust. Retrieved from http://dementia.stir.ac.uk/design/good-practice-guidelines . Homes and Communities Agency (2009). HAPPI: Housing our Ageing Population: Panel for Innovation. Retrieved from http://www.homesandcommunities.co.uk/sites/default/files/happi final report 031209.pdf.

Kelly, F., Innes, A. \& Dincarslan, O. (2011). Improving care home design for people with dementia. Journal of Care Services Management, 5(3), 147- 155.

Kiata, L., Kerse, N M., Hughes, W.E., Hayman, K J., Robertson, M.C., La Grow, S. \& Campbell, A.J. (2008). Agreement and compliance with advice on removing mats or rugs by older people with visual impairments. Journal of Visual Impairment \& Blindness, 102(3), 167-172. 
Kondo, T., Mann, W.C., Tomita, M. \& Ottenbacher, K.J. (1997). The use of microwave ovens by elderly persons with disabilities. American Journal of Occupational Therapy, 51(9), 739-747.

Kwack, H., Relf, P.D. \& Rudolph, J. (2004). Adapting garden activities for overcoming difficulties of individuals with dementia and physical limitations. Activities, Adaptation \& Aging, 29(1), 1-13.

La Grow, S., Robertson, M.C., Campbell, A.J., Clarke, G.A., \& Kerse, N.M. (2006). Reducing hazard related falls in people 75 years and older with significant visual impairment: how did a successful program work? Injury Prevention, 12(5), 296-301.

Lawrence, V., \& Murray, J. (2009). Promoting independent living among people with dementia and sight loss. Journal of Care Services Management, 3(3), 261-274.

Lawrence, V., Murray, J., Ffytche, D., \& Banerjee, S. (2009). "Out of sight, out of mind": a qualitative study of visual impairment and dementia from three perspectives. International Psychogeriatrics / IPA, 21(3), 511-518.

Letts, L., Minezes, J., Edwards, M., Berenyi, J., Moros, K., O'Neill, C. \& O'Toole, C. (2011). Effectiveness of Interventions Designed to Modify and Maintain Perceptual Abilities in People With Alzheimer's Disease and Related Dementias. The American Journal of Occupational Therapy, 65(5), 505-513.

Littlefair, P. (2010). Daylighting and windows in homes of people with sight loss. Thomas Pocklington Trust Research Findings 30. Retrieved from http://www.pocklingtontrust.org.uk/Resources/Thomas\%20Pocklington/Documents/PDF/Research\%20Publications/RF30.pdf (and full report, available on request from Thomas Pocklington Trust).

Long, R.G. (1995). Housing design and persons with visual impairment: Report of focus-group discussions. Journal of Visual Impairment \& Blindness, 89(1), 59-69. 
Marquardt, G. (2011). Wayfinding for people with dementia: a review of the role of architectural design. Herd, 4(2), 75-90.

Martin, S., Kelly, G., Kernohan, W. G., McCreight, B. \& Nugent, C. (2008). Smart home technologies for health and social care support. Cochrane Database of Systematic Reviews, Oct 8(4):CD006412.

McNair, D., Cunningham, C., Pollock, R. \& McGuire, B. (2010). Light and lighting design for people with dementia. Stirling: University of Stirling, Dementia Services Development Centre.

Mihailidis, A. \& Fernie, G.R. (2002). Context-aware assistive devices for older adults with dementia. Gerontechnology, 2(2), 173-188.

Mitchell, L. \& Burton, E. (2010). Designing dementia-friendly neighbourhoods: helping people with dementia to get out and about. Journal of Integrated Care, 18(6), 11-18.

Moore, G.F., Audrey, S., Barker, M., Bond, L., Bonell, C., Hardeman, W., Moore, L., O'Cathain, A., Tinati, T., Wight, D. \& Baird, J. (2015). Process evaluation of complex interventions: Medical Research Council guidance. British Medical Journal 350:h1258 NHS Centre for Reviews and Dissemination (NHS CRD) (2001) 'Undertaking Systematic Reviews of Research on Effectiveness, CRD Report No. 4 (2nd Edition)'. York: NHS Centre for Reviews and Development.

Pawson, R. \& Tilley N. (1997) Realistic Evaluation. London: Sage.

Percival, J. (2007). Lighting the homes of people with sight loss: an overview of recent research. Thomas Pocklington Trust Research Findings 15. Retrieved from http://www.pocklingtontrust.org.uk/research/publications/rf15op13 .

Pollock, R., McNair, D., McGuire, B. \& Cunningham, C. (2007). Designing Lighting for People with Dementia. Stirling: The Dementia Services Development Centre and University of Stirling. 
Rappe, E. \& Topo, P. (2007). Contact with Outdoor Greenery Can Support Competence Among People with Dementia. Journal of Housing for the Elderly, 21(3-4), 229-248.

Torrington, J. \& Lewis, A. (2011). Extra care housing for people with sight loss: lighting and design issues. Thomas Pocklington Trust Research Findings 36. Retrieved from http://www.pocklingtontrust.org.uk/Resources/Thomas\%20Pocklington/Documents/PDF/Research\%20Publications/Research\%2 Ofindings\%2036.pdf (and final report, available on request from Thomas Pocklington Trust).

Unwin, B.K., Andrews, C.M., Andrews, P.M., \& Hanson, J.L. (2009). Therapeutic home adaptations for older adults with disabilities. American Family Physician, 80(9), 963.

Utton, D. (2009). The design of housing for people with dementia. Journal of Care Services Management, 3(4), 380-390.

van Hoof, J., Kort, H., van Waarde, H. \& Blom, M.M. (2010). Environmental Interventions and the Design of Homes for Older Adults With Dementia: An Overview. American Journal of Alzheimer's Disease and Other Dementias, 25(3), 202-232.

Zwijsen, S A., Niemeijer, A.R. \& Hertogh, C.M.P.M. (2011). Ethics of using assistive technology in the care for community-dwelling elderly people: An overview of the literature. Aging \& Mental Health, 15(4), 419-427. 